\title{
Low Dimensional Lattice Diffusion in Solids Investigated by Nuclear Spin Echo Measurements
}

\author{
Naoki Asakawa * Kiyohiko Matsubara, and Yoshio Inoue \\ Department of Biomolecular Engineering, Tokyo Institute of Technology. 4259 Nagatsuta-cho, \\ Midori-ku, Yokohama, Kanagawa 226-8501, Japan
}

(Dated: November 20, 2018)

\begin{abstract}
Lattice diffusion and internal local magnetic field gradients in solids are investigated by numerical simulation of nuclear mangetic resonance(NMR) spin echo experiments. The Fourier-spectrum method is employed in order to solve the Bloch-Torrey equation with arbitrary magnetic field gradients in one- and two-dimensional lattice restrictions.
\end{abstract}

Keywords: NMR, spin echo, low dimensional diffusion, local magnetic field gradient

\section{INTRODUCTION}

Transport phenomena are often encountered in solutions, fluids, gels, and quasi particles associated with elementary excitations in solids, and so on. In particular, low dimensional transport phenomena are received much attraction in various research fields of electronic devices such as polymeric conducting materials 1 , quasi one-dimensional organic conductors ${ }^{2}$ and rechargeable batteries 3.4 . In these matarials or devices, low dimensional diffusive or hopping motion such as charged ions, defects, quasi-particles(e.g., exciton, polaron, conformon) in their crystalline lattices plays an important role in origins of their physical properties. Some of such molecular motions in crystalline solids are called "lattice diffusion," which is defined as transport phenomena of (quasi-)particles of which positions are restricted in lattice points in crystals. The motion often leads to motional averaging of local magnetic fields in solids. Nuclear magnetic resonance(NMR) spectroscopy is one of promising nondestructive methods for investigating these phenomena. Several attempts have recently been reported to make NMR measurements of ultra slow diffusion in solids 5.6 .12 .

Among these, the pulse field gradient(PFG) technique with strong magnetic field gradient(MFG) has been given much attention for detection of such diffusions. However, the PFG technique involves some problems in cases, which are (i) attenuation of sensitivity in association of application of PFG, (ii) a lack of magnitude of PFG for ultra slow diffusion measurements, and (iii) the case that the system has large internal $\operatorname{MFG}\left(G_{\mathrm{i}}\right)$ compared to external PFG and/or large heterogeneous MFG.

The problem (i) and (ii) are often pronounced in molecular diffusion measurements. Since the problem (iii) is not problematic in cases of systems with large diffusion coefficient, it has often been ignored. In such cases, the internal MGF is thought to be averaged to zero, and then effects of the internal MFG will be small or negligible. However, in solids, the diffusion coefficient can be quite small and a local internal MFG, which in some cases heterogeneous and/or anisotropic, can be remained ${ }^{12}$.

In such situations, an effective MFG is no longer the same as the external $\operatorname{MFG}\left(G_{\mathrm{e}}\right)$, which is likely to mask the accurate diffusion coefficient. Even in the case that the system has large local MFG, it will not be problematic if the root mean squared displacement by diffusion $(\sqrt{D t})$ is much larger than the periodicity of the local MFG, because the local MFG is expected to be averaged out during diffusion. On the contrary, in the case of systems with small diffusion coefficient, the local MFG can be remained and spin echo trains of magnetic resonance can be further attenuated by the local MFG as well as by the external MFG.

So far, there are several kinds of possible approaches to cancel the effect of local MFG, that is to set up the experimental condition of $G_{\mathrm{e}} \gg G_{\mathrm{i}}$ by production of large external MFG by an anti-Helmholtz superconducting magnet or a fringe field of a superconducting magnet. By using anti-Helmholtz superconducting magnet, one can obtain a MFG up to $200 \mathrm{~T} \cdot \mathrm{m}^{-16}$. On the other hands, by using superconducting fringe field, one can obtain up to $60 \mathrm{~T} \cdot \mathrm{m}^{-15}$. There are the other approaches to cancel local MFG by using bipolar gradients 7.8 .9 .10 or rotary echos 11 . These methods are based on the idea of cancelation of unwanted local MFG. On the contrary, we are interested in local MFG, which is thought to be characteristic to each systems particularly to solids, and it should be closely related to the molecular and electronic structure.

There are a number of literatures available to calculate spin echos in restricted geometries $13,14,15,16,17$. Most of these researches are devoted to describe pulse gradient spin echo(PGSE) experiments. The spin echo experiment under a static field gradient is a kind of niche applications, and its theoretical treatment, namely, solvation of Bloch-Torrey equation ${ }^{18}$, is still lacking. Up to now, Axelrod and Sen have shown a solution of one dimensional Bloch-Torrey equation under the linear gradient condition by using an eigen mode technique ${ }^{17}$. In this Letter, we shall extend their treatment to spin echo experiments with two-dimensional lattice diffusion under arbitrary magnetic field gradient shape. Although the general theory of spectral density function for twodimensional lattice diffusion is developed ${ }^{19}$, effects of $2 \mathrm{D}$ diffusion on nuclear spin echo experiments have not been investigated so far.

The method developed here will be useful to inves- 
tigate transport phenomena in low dimensional solids, such as movement of lattice defects(including soliton in disordered solids), dynamics of elementary excitation(including exciton and polaron), transport of charge carriers, and so on. Further, recent developments on magnetic resonance force microscopy(MRFM) show that one can perform in situ imaging with a scale of several tens of nanometers, where ultra high magnetic field gradients of over $10^{3} \sim 10^{4} \mathrm{~T} / \mathrm{m}$ are generally used 20.21 . Up to now, there are no publications available to obtain spin-echo measurements under a condition of such a huge MFG, which can be comparable to local magnetic field gradient with atomic scale. The theory presented in this Letter will be useful for analyses of such experiments as well.

\section{THEORETICAL}

The method presented here is based on the numerical solution of the Bloch-Torrey equation by the Fourierspectrum method 22 . Axelrod and Sen have proposed the methodology for calculating amplitudes of spin echos for a nucleus diffusing under a linearly inhomogeneous magnetic field condition 17 . Although they have shown the solution with a linear magnetic field gradient by an eigen mode expansion method, the details of the numerical procedure was not shown. In this article, we shall demonstrate a mathematical formulation of solvation of the Bloch-Torrey equation by the Fourier-spectrum method and explore spin echo amplitudes under various magnetic field gradients.

So far, we have attempted to solve the differential equation of diffusion equation in the real space by CrankNicolson method 23 . However, we have suffered from the underflow of variables due to iterative multiplication in many times, of the time evolution operator for the spin echo experient. Furthermore, while the Crank-Nicolson method preserves the absolute stability against the size of each step in the real space, we observed the strong dependence of spin echo amplitudes on magnitude of interval of time step.

Thus, these problems lead us to motivation for numerical simulation in the Fourier space concerning coordinates in real space.

Below we shall describe the mathematical formulation for Bloch-Torrey equation with magnetic field gradient of an arbitrary wave form.

\section{A. Solution for 1D Bloch-Torrey Equation with arbitrary magnetic field gradient}

The Bloch-Torrey equation is shown in the following:

$$
\frac{\partial M}{\partial t}=\tilde{D}_{0} \frac{\partial^{2} M}{\partial \tilde{x}^{2}}-i \tilde{\gamma} f(\tilde{x}) M
$$

where the tilde denotes dimensionless parameter in order the problem to be treated as general. The effects of longitudinal relaxation $\left(T_{1}\right)$ and intrinsic transverse relaxation $\left(T_{2}\right)$ in original Bloch-Torrey equation was neglected in the calculation. But the effect must of course be taken into account in practical applications. Dimensionless diffusion coefficient $\left(\tilde{D}_{0}\right)$, dimensionless gyromagnetic $\operatorname{ratio}(\tilde{\gamma})$, and one-dimensional coordinate $(\tilde{x})$ are defined as

$$
\begin{aligned}
\tilde{D}_{0} & =\frac{D_{0} \tau}{L_{s}} \\
\tilde{\gamma} & =\tau g \gamma L_{s} \\
\tilde{x} & =\frac{x}{L_{s}} .
\end{aligned}
$$

$f(\tilde{x})$ is a functional form of $1 \mathrm{D}$ MFG.

Let us think the expansion of the Eq.(11) by a series of orthogonal functions, $\Psi_{k}(\tilde{x})$.

$$
\mathcal{F}\left[\frac{\partial M}{\partial t}\right]=\tilde{D}_{0} \mathcal{F}\left[\frac{\partial^{2} M}{\partial \tilde{x}^{2}}\right]-i \tilde{\gamma} \mathcal{F}[f(\tilde{x}) M] .
$$

Here, we used linearity of orthogonal function transformation. The second term of the right hand side of Eq.(5) is obtained by convolution:

$$
\mathcal{F}[f g]=\mathcal{F}[f] * \mathcal{F}[g]
$$

Thus, one can write down the practical form of Eq. (1) like the following:

$$
\frac{d M_{k}(t)}{d t}=-(\pi k)^{2} \tilde{D}_{0} M_{k}(t)-i \tilde{\gamma} \sum_{p=0}^{N-1} V_{k-p} M_{p}(t),(7)
$$

Here,

$$
M_{k}(t)=\mathcal{F}[M]=\int_{-1 / 2}^{1 / 2} M(\tilde{x}, t) \Psi_{k}(\tilde{x}) d \tilde{x} .
$$

$V_{k}$ is a $\mathrm{k}$-th coefficient for orthogonal function transform(for example, Fourier transform) of $\tilde{x}$ :

$$
V_{k}=\int_{-1 / 2}^{1 / 2} f(\tilde{x}) \Psi_{k}(\tilde{x}) d \tilde{x}
$$

Further, the first term of the right hand side of Eq.(7) can be obtained by using the fact that second derivative in real space corresponds to the multiplication with $k^{2}$.

$$
\frac{d M_{k}(t)}{d t}=(\hat{W}-i \tilde{\gamma} \hat{B}) M_{k}(t)
$$

Here,

$$
\begin{gathered}
\hat{W}_{k l}=\left\{\begin{array}{l}
-(\pi \cdot k)^{2} \tilde{D}_{0} \text { if } k=l \\
0 \text { if } k \neq l
\end{array}\right. \\
\hat{B}_{k l}=V_{k-l}
\end{gathered}
$$


Let us think a basis set with taking account of the boundary condition of $\tilde{x}:[-1 / 2: 1 / 2]$. Shown below is the eigen modes using sinusoidal(only cosine components) orthogonal functions for the Laplacian, $\tilde{\Delta}_{0}=-\tilde{\nabla}^{2}$, of the one dimensional diffusion equation,

$$
\Psi_{k}(\tilde{x})=C_{k} \cos \left(\pi k\left(\tilde{x}+\frac{1}{2}\right)\right)
$$

where

$$
C_{k}= \begin{cases}1 & \text { if } k=0 \\ \sqrt{2} & \text { if } k \neq 0\end{cases}
$$

and corresponding eigen values are $\tilde{\lambda}_{k}=(\pi k)^{2}$.

Since it is unnecessary to think the time ordering during the echo time, $\tau$, in transverse relaxation time measurements, one can take $\tau$ as one step.

The time evolution operator for the echo time, $\tau$, can be defined as

$$
U_{+}=e^{(\hat{W}-i \tilde{\gamma} \hat{B}) \tau}
$$

Thus, the propagator for the two pulse Hahn echo ${ }^{24}$ can be express as

$$
U_{2 \tau}=U_{-} U_{+}=\left(U_{+}\right)^{*} U_{+} .
$$

Similarly, that for the second echo of Carr-Purcell Meiboom-Gill(CPMG) ${ }^{25,26}$ can be expressed as

$$
U_{4 \tau}=U_{+} U_{-} U_{-} U_{+},
$$

and that for n-th echo of CPMG,

$$
U_{2 n \tau}=U_{2 \tau}^{\bmod (n, 2)} U_{4 \tau}^{\text {floor }(n, 2)} .
$$

Therefore, The k-th Fourier component of the magnetization at a time, $t=2 n \tau$, can be calculated by

$$
M_{k}(t)=U_{2 n \tau} M_{k}(0)
$$

Here, the initial magnetization, $M_{k}(0)$, can be obtained by using the initial condition of $\phi(\tilde{x})$ :

$$
\begin{aligned}
\sum_{k=0}^{\infty} M_{k}(0) \Psi_{k}(\tilde{x}) & =\sum_{k=0}^{\infty} M_{k}(0) \cos \left(\pi k\left(\tilde{x}+\frac{1}{2}\right)\right) \\
& \equiv \phi(\tilde{x})
\end{aligned}
$$

The Fourier component, $M_{k}(0)$, of the initial magnetization, can be calculated by inverse Fourier transform of $\phi(\tilde{x})$. For example, in the case of homogeneous spin density, namely, in the case of the initial condition of $\phi(\tilde{x})=1$ (constant), all the components are zero except $\mathrm{k}=0$ since the inverse Fourier transform is a $\delta$-function. Substituting the obtained $M_{k}(0)$ into Eq. (19), the kth magnetization, $M_{k}(t)$, at a time, $t(=2 n \tau)$, can be computed. Finally, one can calculate the magnetization, $M(t)$ by inverse Fourier transform of $M_{k}(t)$ and the summation of each components which correspond to each components in real space;

$$
M(t)=\sum_{\tilde{x}=-1 / 2}^{1 / 2} \mathcal{F}^{-1}\left[M_{k}(t)\right]
$$

All the figures in this article are plots of the relaxation exponent, $\log \left|\frac{M(0)}{M(t=2 n \tau)}\right| / n$, as a function of $\tilde{D}_{0}$.

\section{B. Two-dimensional Diffusion}

We extended the 1D Bloch-Torrey equation to the case of anisotropic 2D lattice diffusion. For anisotropic 2D diffusion, the Bloch-Torrey equation is expressed as follows.

$$
\frac{\partial M}{\partial t}=\tilde{D}_{\tilde{x} 0} \frac{\partial^{2} M}{\partial \tilde{x}^{2}}+\tilde{D}_{\tilde{y} 0} \frac{\partial^{2} M}{\partial \tilde{y}^{2}}-i \tilde{\gamma} f(\tilde{x}, \tilde{y}) M .
$$

The solution can be written as

$$
M(t)=U_{2 n \tau} M(0)
$$

where

$$
U_{2 n \tau}=\exp \left(-\int_{0}^{2 n \tau}\left(-D_{0} \nabla^{2}-i \gamma \hat{f}(\tilde{x}, \tilde{y})\right) d t\right)
$$

Now we perform Fourier transform using the series of two dimensional orthogonal functions, $\Psi_{m n}(\tilde{x}, \tilde{y})$,

$$
\begin{aligned}
\Psi_{m n}(\tilde{x}, \tilde{y}) & =C_{m n} \Psi_{m}(\tilde{x}) \Psi_{n}(\tilde{y}) \\
& =C_{m n} \cos \left(\pi m\left(\tilde{x}+\frac{1}{2}\right)\right) \cos \left(\pi n\left(\tilde{y}+\frac{1}{2}\right)\right)
\end{aligned}
$$

$C_{m n}$ is a normalization constant and $m, n$ are eigen mode values.

$$
C_{m n}= \begin{cases}1 & (m=n=0) \\ \sqrt{2} & (m=0 \text { or } n=0) \\ 2 & (m \neq 0 \text { and } n \neq 0)\end{cases}
$$

Let us consider the space Fourier transform of Eq.(22):

$$
\mathcal{F}\left[M_{t}\right]=D_{0} \mathcal{F}\left[\nabla^{2} M\right]-i \gamma \mathcal{F}[f(\tilde{x}, \tilde{y}) M] .
$$

Again, $\mathcal{F}[M]$ stands for the 2D Fourier transform of magnetization $M$,

$$
\begin{aligned}
\hat{M}_{m n}(t) & =\mathcal{F}[M] \\
& =\iint_{-\frac{1}{2}}^{\frac{1}{2}} M(\tilde{x}, \tilde{y}, t) \Psi_{m n}(\tilde{x}, \tilde{y}) d \tilde{x} d \tilde{y}
\end{aligned}
$$

Here, $\hat{M}$ represents the magnetization in the Fourier space. We begin by the procedure for calculating $\mathcal{F}\left[\nabla^{2} M\right]$ of Eq.(27).

$$
\mathcal{F}\left[\nabla^{2} M\right]=\mathcal{F}\left[M_{\tilde{x} \tilde{x}}\right]+\mathcal{F}\left[M_{\tilde{y} \tilde{y}}\right]
$$


For the second-order derivative of the $\tilde{y}$ component,

$$
\begin{aligned}
& \mathcal{F}\left[M_{\tilde{y} \tilde{y}}\right] \\
& =\left(\begin{array}{cccc}
-(\pi \cdot 0)^{2} M_{00} & -(\pi \cdot 1)^{2} M_{01} & \ldots & -(\pi \cdot n)^{2} M_{0 n} \\
-(\pi \cdot 0)^{2} M_{10} & -(\pi \cdot 1)^{2} M_{11} & \ldots & -(\pi \cdot n)^{2} M_{1 n} \\
\vdots & \vdots & \ddots & \vdots \\
-(\pi \cdot 0)^{2} M_{m 0} & -(\pi \cdot 1)^{2} M_{m 1} & \ldots & -(\pi \cdot n)^{2} M_{m n}
\end{array}\right) \\
& =\left(\begin{array}{cccc}
M_{00} & M_{01} & \ldots & M_{0 n} \\
M_{10} & M_{11} & \ldots & M_{1 n} \\
\vdots & \vdots & \ddots & \vdots \\
M_{m 0} & M_{m 1} & \ldots & M_{m n}
\end{array}\right) \\
& \times\left(\begin{array}{cccc}
-(\pi \cdot 0)^{2} & 0 & \cdots & 0 \\
0 & -(\pi \cdot 1)^{2} & \cdots & 0 \\
\vdots & \vdots & \ddots & \vdots \\
0 & 0 & \ldots & -(\pi \cdot n)^{2}
\end{array}\right) \\
& =\hat{M} \hat{W}_{y}
\end{aligned}
$$

Similarly, for the $\tilde{x}$ component, $\mathcal{F}\left[M_{\tilde{x} \tilde{x}}\right]=\hat{W}_{x} \hat{M}$, where,

$$
\hat{W}_{x}=\left(\begin{array}{cccc}
-(\pi \cdot 0)^{2} & 0 & \ldots & 0 \\
0 & -(\pi \cdot 1)^{2} & \ldots & 0 \\
\vdots & \vdots & \ddots & \vdots \\
0 & 0 & \ldots & -(\pi \cdot m)^{2}
\end{array}\right)
$$

Thus,

$$
\mathcal{F}\left[M_{\tilde{x} \tilde{x}}\right]+\mathcal{F}\left[M_{\tilde{y} \tilde{y}}\right]=\hat{W}_{x} \hat{M}+\hat{M} \hat{W}_{y}
$$

When $m=n$, both $\hat{W}_{x}$ and $\hat{W}_{y}$ are same diagonal matrices each other, so we can rewrite,

$$
\mathcal{F}\left[M_{\tilde{x} \tilde{x}}\right]+\mathcal{F}\left[M_{\tilde{y} \tilde{y}}\right]=\hat{W}_{x} \hat{M}+\hat{M} \hat{W}_{x}
$$

Now we introduce a Laplacian superoperator $\hat{\hat{W}}$ based on commutator superoperators 27 .

$$
\begin{aligned}
\hat{\hat{W}} \hat{M} & =\hat{W}_{x} \hat{M}+\hat{M} \hat{W}_{x} \\
& =\hat{W}_{x} \hat{M} \times E+E \times \hat{M} \hat{W}_{x}
\end{aligned}
$$

where $E$ represents the unity operator. This leads to the matrix representation,

$$
\hat{\hat{W}}=\hat{W}_{x} \otimes \hat{E}+E \otimes \tilde{W}_{x}
$$

where $\tilde{W}_{x}$ represents the transposed matrix of $\hat{W}_{x}$. $\otimes$ denotes direct product of matrices.

In the practical calculation, we assume the square lattice and adopt the identical number of eigen modes for $\tilde{x}$ and $\tilde{y} \operatorname{dimensions}(m=n)$, and $\tilde{E}=E$.

$$
\mathcal{F}\left[\nabla^{2} M\right]=\hat{\hat{W}} \hat{M}
$$

Now we go on to show the calculation of the second term, $\mathcal{F}[f(\tilde{x}, \tilde{y}) \mathbf{M}]$, in Eq.(27). The Fourier transformation of this term can be rewritten using a convolution,

$$
\begin{aligned}
\mathcal{F}[f(\tilde{x}, \tilde{y}) M] & =\mathcal{F}[f(\tilde{x}, \tilde{y})] * \mathcal{F}[M] \\
& =\sum_{p} \sum_{q} V_{m-p, n-q} M_{p, q},
\end{aligned}
$$

where $V_{m, n}$ represents $(m, n)$-th component of Fourier space of $f(x, y)$.

$$
V_{m, n}=\iint_{-\frac{1}{2}}^{\frac{1}{2}} f(x, y) \Psi_{m n}(x, y) d x d y
$$

Thus, the convolution superoperator, $\hat{\hat{B}}$, between $\mathcal{F}[f(\tilde{x}, \tilde{y})]$ and $\mathcal{F}\left[M_{m n}\right]$ is defined as

$$
\mathcal{F}[f(\tilde{x}, \tilde{y}) M]=\mathcal{F}[f(\tilde{x}, \tilde{y})] * \mathcal{F}[M] \rightarrow \hat{\hat{B}} \hat{\hat{M}}
$$

Here, it is worth to note that the computational effort of the convolution in Eq. [37] is proportional to $\mathcal{O}\left(\mathrm{m}^{2} \times\right.$ $n^{2}$ ), so this calculation becomes very huge. To avoid this difficulty, one can employ the transform method utilizing fast-Fourier Transform (FFT) in oder to diminish the computational time $\mathrm{e}^{22}$. The computational effort of the transform method is proportional to $\mathcal{O}(m \log m \times n \log n)$. In the transform method, one calculate the elaborated formula:

$$
\mathcal{F}\left[\mathcal{F}^{-1}\left[f_{m n}\right] \times \mathcal{F}^{-1}\left[M_{m n}\right]\right] .
$$

By the virtue of fast Fourier Transform(FFT) algorithm, one can reduce the number of multiplication to calculate convolution. Unfortunately, we could not find the superoperator for the convolution, then, we implemented the direct calculation of convolution by using a parallel programming technique of the Message Passing Interface(MPI) standard.

\section{RESULTS AND DISCUSSION}

\section{A. Comparison with the result of the second-order cumulant expansion}

Fig 1 shows a comparison of the numerical solution of Bloch-Torrey equation by the Fourier spectrum method, with the results of the second-order cumulant expansion(so-called gaussian phase approximation). The calculation was performed for the cases of a linear $(f(x)=$ $x)$ and parabolic $\left(f(x)=4 x^{2}-0.5\right)$ magnetic field gradients in one-dimensional. According to Axelrod and Sen, the second cumulant expansion become a good approximation for both the short time and motionaly averaging regimes ${ }^{17}$. For the linear case, we obtained the exactly same behavior on the result of Fourier spectral method as the result by Axelrod and Sen 17 . As for the localized regime, the relaxation exponent indicates strong dependence on the functional shape of a magnet field gradient. 


\section{B. Effect of Dimensionality of Diffusion}

A sinusoidal function may be a good candidate as a first step, for the expression of internal magnetic field gradient in a crystal. For example, the Peierls potential is often approximated by the sinusoidal. Fig 2 shows the decay exponentials for the 8th CPMG echos under the condition of the sinusoidal gradients, shown in the figure, as a function of dimensionless diffusion coefficient, $\tilde{D}_{0}\left(\tilde{D_{0}}=\tilde{D_{x 0}}\right.$ for one-dimensional cases and $\tilde{D}_{0}=\left({\tilde{D_{x 0}}}^{2}+{\tilde{D_{y 0}}}^{2}\right)^{1 / 2}$ for two-dimensional cases $)$. We took into account the lowest eigen modes up to 16 th and used the $\tilde{\gamma}$ value of 10 . For the so-called localization regime, the lower attenuation of decay exponential was observed for the $2 \mathrm{D}$ diffusion case than that for the $1 \mathrm{D}$ case. It is also found that the dimensionality of diffusion is insensitive both at the short time and at the motional averaging regimes. At the motional averaging regimes, although a slight increase of echo attenuation occurs on $2 \mathrm{D}$ cases, the decay exponent as a function of $\tilde{D}_{0}$ shows the same slope irrespective of dimensionality of diffusion.

\section{Effect of Anisotropic Diffusion in a 2D lattice}

Now let us look at a situation that a system of interest shows the anisotropic diffusion from internal and/or external reasons. For example, putting the sample in the external electric field corresponds to the latter case. Fig 3 shows the effect of anisotropy of diffusion on CPMG decay exponent. One observe a double maximum profile of relaxation exponent for diffusion with an anisotropy of over 200. Two peaks of the relaxation exponent have some interesting features.

First, while the main peak around the $\tilde{D}_{0}$ value of unity does not nearly move its position with respect to changes in anisotropy, the second peak moves to positions with larger $\tilde{D}_{0}$ values proportional to the anisotropy. Second, the ratio of the relaxation exponent at two maxima is almost constant with respect to changes in the anisotropy of diffusion, but the ratio and the shape of peak are strongly dependent to functional form of the field gradient. Third, the differences in dimensionality of diffusion show much larger effect on relaxation exponent than functional form of MFG. By comparing Fig [3] and b) with c) and d), one can realize that the relaxation exponents for two-dimensional MFG are much larger than those for one-dimensional for the case of isotropic diffusion $\left(D_{x 0} / D_{y 0}=1\right)$.

From the above results, it would be possible to determine the functional form of MFG by transverse relaxation time measurements at localization regime when the anisotropy of diffusion is large. If the MFG is originated from internal one, for example, crystalline periodicity, Peierls potential ${ }^{28,29.30}$, Friedel oscillation ${ }^{31}$, and so on, transverse relaxation time measurements will become a novel tool for determination of the functional form of these internal properties.

\section{CONCLUSION}

We have explored diffusion and internal local magnetic field gradients in solids by nuclear magnetic resonance(NMR) transverse relaxation time measurements. The Fourier-spectrum method was employed in order to solve the Bloch-Torrey equation with arbitrary magnetic field gradients in one- and two-dimensional lattice restrictions. If there are no phase transitions or deformations of samples for a temperature region interested, variable temperature CPMG measurements with a fixed echo time have a possibility for determination of dimensionality of diffusion processes found in the samples. The method will be applicable to several research areas such as transport phenomena of charge carriers in ion conducting materials, fluid dynamics in low dimensional porus materials, and dynamics of low dimensional elementary excitations in solids.

\section{Acknowledgments}

This work is supported by Ministry of Education, Culture, Sports, Science and Technology(Japan) through a Grant-in-Aid for science research(No.16685012).
* Corresponding Author; Electronic address: nasakawa@bio.titech.ac.jp

1 M.Nechtschein, F.Devreux, R.L.Greene, T.C.Clarke, and G.B.Street, Phys. Rev. Lett., 44(1980) 356.

2 R.Ruf, N.Kaplan, and E.Dormann, Phys. Rev. Lett., 74(1995)2122.

3 A.F.McDowell, C.F.Mendelsohn, M.S.Conradi, R.C.Bowman, Jr., and A.J.Maeland, Phys. Rev., B51(1995)6336.

4 F.Kimmerle, G.Majer, U.Kaess, A.J.Maeland, M.S.Conradi, and A.F.McDowell, J. Alloys. Compounds, 264(1998)63.
5 R.Kimmich, W.Unrath, G.Schnur, and E.Rommel, J. Magn. Reson., 91(1991)136.

6 I.Chang, G.Hinze, G.Diezemann, F.Fujara, and H.Sillescu, Phys. Rev. Lett., 76(1996)2523; I.Chang, F.Fufara, B.Geil, G.Hinze, H.Sillescu, A.Tölle, J. Non-Crystalline Solids, 172-174(1994)674.

7 R.F.Karlicek and I.J.Lowe, J.Magn.Reson., 37(1980)75.

8 R.M.Cotts, M.J.R.Hoch, T.Syn, and J.T.Marker, J.Magn.Reson., 83(1989)252.

9 E.J.Fordham, S.J.Gibbs, L.D.Hall, Magn.Reson.Imag., 12(1989)279.

10 D.Wu, A.Chen, C.S.Johnson,Jr., J.Magn.Reson., 
A115(1995)260.

11 I.Ardelean and R.Kimmich, Annual Repts. NMR Spectroscopy, 49, 43(2003).

12 D.C.Ailion, Solid State Ionics, 125(1999)251.

13 A.Caprihan, L.Z.Wang, and E.Fukushima, J. Magn. Reson., A118(1996)94.

14 D.Sheltraw and V.M.Kenkre, J. Magn. Reson., A122(1996)126.

15 P.T.Callaghan, J. Magn. Reson., 129(1997)74.

16 A.V.Barzykin, Phys. Rev., B58(1998)14171.

17 S.Axelrod and P.N.Sen, J. Chem. Phys., 114(2001)6878.

18 H.C.Torrey, Phys. Rev., 104(1956)563.

19 P.C.L.Stephenson and C.A.Sholl, J. Phys.:Condens. Matter, 5(1993) 2809.

${ }^{20}$ K.J.Bruland, W.M.Dougherty, J.L.Garbini, J.A.Sidles, and S.H.Chao, Appl. Phys. Letts., 73(1998)3159.

21 S.H.Chao, W.M.Dougherty, J.L.Garbini, and J.A.Sidles, Rev. Sci. Instrum., 75(2004)1175.

22 J.P.Boyd, Chebyshev and Fourier Spectrum Methods, Second ed., Dover, New York, 2001.

23 W.F.Ames, Numerical Methods for Partial Differential Equations, 2nd ed., Academic Press, New York, 1977.

24 E.L.Hahn, Phys. Rev., 80(1950)580.

25 H.Y.Carr and E.M.Purcell, Phys. Rev., 94(1954)630

26 S.Meiboom and D.Gill, Rev. Sci. Instrum., 29(1958)688.

27 R.R.Ernst, G.Bodenhausen, and A.Wokaun, Principle of Nuclear Magnetic Resonance in One and Two Dimension, Oxford Press, Oxford, 1987.

28 B.Barbara, J. Phys., 34(1973)1039.

29 H.R.Hilzinger and H.Kronmuller, Phys. Stat. Solidi, $\mathbf{5 4}(1986) 593$.

30 K.S.Novoselov, A.K.Geim, S.V.Dubonos, E.W.Hill, and I.V.Grigorieva, Nature, 426(2003)812.

31 I.Yu, J. Magn. Reson., A104(1993)209.

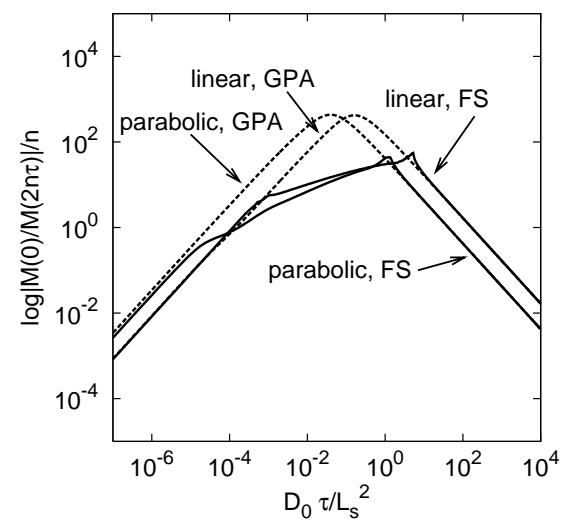

FIG. 1: Comparison of numerical solution of 1D Bloch-Torrey equation by the Fourier spectrum(FS) method, with the results of the second-order cumulant expansion(gaussain phase approximation;GPA). The calculation was performed for the cases of linear $(f(x)=x)$ and parabolic $\left(f(x)=4 x^{2}-0.5\right)$ magnetic field grandients. The lowest eigen modes up to 64 th were taken into account.

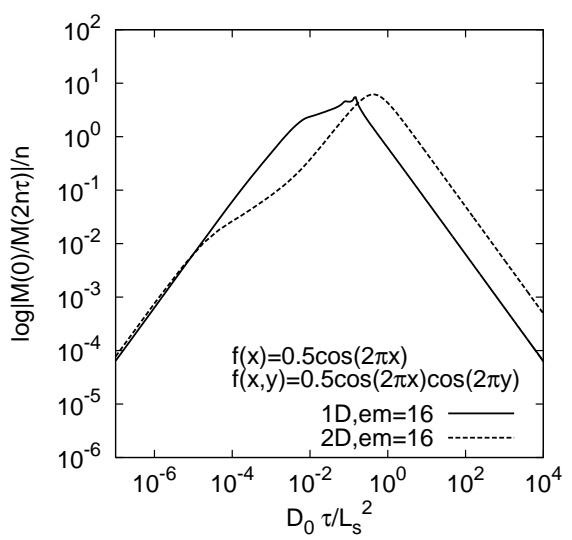

FIG. 2: Effect of dimensionality of diffusion on decay exponent of CPMG spin echo experiments. The eigen modes up to 16 th is taken into account and the value of $\tilde{\gamma}$ is set at 10 .
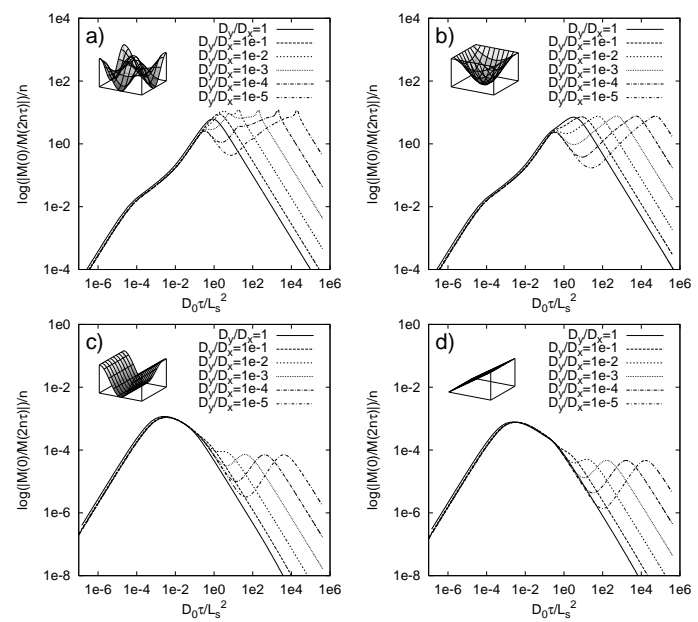

FIG. 3: Effect of anisotropic diffusion on decay exponent of CPMG spin echo experiments. a) $f(x, y)=$ $0.5 \cos (2 \pi x) \cos (2 \pi y)$, b) $f(x, y)=0.5-\cos (\pi x) \cos (\pi y), \mathrm{c})$ $f(x, y)=-0.5 \cos (2 \pi x)$, and d) $f(x, y)=x$. 